\title{
Assessment method of harmonic emission level based on pan-normal harmonic impedance cloud
}

\author{
Zhao Guosheng ${ }^{1, a}$, Zhao Dan ${ }^{1, b}$ and Qiu Siyu ${ }^{2, c}$ \\ ${ }^{1}$ Zhengzhou university, China \\ ${ }^{2}$ Sichuan university, China \\ azgs410@zzu.edu.cn ,b340654733@qq.com, csiyu_qiu@foxmail.com
}

Keywords: Power quality, harmonic impedance, harmonic emission level, pan-normal, impedance cloud, filtering threshold.

Abstract. In this paper, a method for estimating the system harmonic impedance and harmonic emission level based on the pan-normal impedance cloud is presented. First of all, based on the basic principle of fluctuation method, it can be obtained that the key to estimate the system harmonic impedance is how to identify fluctuation ratio samples closer to the true value through theoretical analysis; Secondly, through the study of the probability distribution of fluctuation ratio, this paper proposes the characteristic of the pan-normal distribution meets the applicable conditions of normal cloud model, which can be used to calculate the digital features of impedance cloud by backward cloud generator; Finally, based on the " $3 E n$ ' Rules" of cloud model, the filtering threshold of impedance is determined, then the system harmonic impedance and harmonic emission level can be estimated by filtering qualified samples. It proves the accuracy and effectiveness of the proposed method by the simulation of theoretical model and calculation of practical engineering examples.

\section{Introduction}

In the modern power system, due to the large number of nonlinear characteristics and impact loads connected to the grid, the problem of serious harmonic pollution has caused the attention of scholars both at home and abroad. The harmonic emission level of the power system side and the user side is usually considered as an indicator for harmonic pollution in the point of common coupling (PCC), so it is very important to assess the harmonic emission level ${ }^{[1,2]}$. The harmonic voltage of PCC is impacted by harmonic source both the system side and user side, how to obtain the harmonic information from the system side and the user side and to determine the contribution of two sides of the harmonic voltage from the harmonic data of PCC, which is difficult to assess the harmonic emission level.

Through theoretical study and derivation, the domestic and foreign scholars put the estimation of harmonic emission level to the system harmonic impedance mostly ${ }^{[3]}$, which are mainly divided into "intervention" and "non- intervention" two categories. And the "intervention" method is mainly through the artificial application of the required signal or change the line operation mode, the change in the operating state of the power system may be a security risk, so the method is not commonly used. The "non-intervention" is the most important method to estimate the system harmonic impedance, mining the useful information from the PCC to estimate the harmonic impedance though various mathematical methods, including the linear regression method ${ }^{[3]}$ and the fluctuation method ${ }^{[2]}$. The linear regression method is used to solve the regression equation directly by using the harmonic data obtained from the PCC, and the system harmonic impedance is obtained by regression analysis, but this method requires a good stability harmonic background over a longer period of time, in order to ensure the accuracy of the regression model. Otherwise, the fluctuation of the background harmonics can cause the instability of the regression model, and the error is large; fluctuation method is derived from the formula of the system harmonic impedance from the Norton equivalent circuit, considering the ratio of the harmonic voltage and current fluctuations in the PCC can be approximated as the system harmonic impedance, so it is widely used. Since the harmonic current of the user and the system side are often simultaneously volatility, the key to improve the accuracy of the fluctuation 
method is that fluctuation ratio samples closer to the true value can be identified. Literature[3] proposed a method for determining the value of the real part of the symbol fluctuation ratio based. The results can not be determined which side is closer through the symbol of the real part of the fluctuation ratio, and the filtering method is limited.

First of all, based on the basic principle of fluctuation method, it can be obtained that the key to estimate the system harmonic impedance is how to identify fluctuation ratio samples closer to the true value through theoretical analysis; Secondly, through studying the probability distribution of fluctuation ratio, this paper finds that the pan-normal distribution characteristic of fluctuation ratio meets the applicable conditions of normal cloud model so that the digital features of impedance cloud can be calculated by backward cloud generator; Finally, based on the " $3 E n$ ' Rules"[4] of cloud model, the filtering threshold of impedance can be obtained, then the system harmonic impedance and harmonic emission level can be estimated by filtering qualified samples. Through the simulation of theoretical model and calculation of practical engineering examples, it proves the accuracy and effectiveness of the proposed method.

\section{Introduction to Cloud Model}

Definition of Cloud. Cloud model originally proposed by academician Li Deyi and others, used to describe the problem between the qualitative and quantitative transformation model uncertainty, and It is a mathematical expression, which is fuzzy and random in objective things or scientific knowledge, with generally usability. Its definition is as follows:

Quantitative $Q$ is determined by the exact value, and $N$ is a qualitative concept corresponding to $Q$, assume that quantitative value $x \in Q$, and $x$ is a random realization of $N$ on $Q . \omega(x) \in[0,1]$ is to determine the degree of $X$ to $N$, and is a stable tendency of the random number $\omega: Q \rightarrow[0,1] \forall x \in Q$, then $x \rightarrow \omega(x)$ distributed over the $Q$ is called a cloud, and each $x$ called cloud droplets ${ }^{[5]}$.

Digital Features of Cloud.A cloud distribution can be described by expected value $E x$, entropy $E n$, and hyper entropy $\mathrm{He}$ three index, which called cloud digital features.

Ex expressed expectation distribution of cloud droplets in the clouds, is a qualitative concept into quantitative theory embodied directly after macro domain; En, the variance of droplet distribution, is a uncertain digital characterization when qualitative concept is quantified, the fuzzy concept in the description of the range of values, reflecting the qualitative concept of fuzzy edge margin; $\mathrm{He}$ is a digital characterization of entropy itself of uncertainty, to reflect the sparse degree of droplet distribution in the clouds, which can further reflect the thickness of clouds, if the hyper entropy is lager, the distribution of cloud droplets in the clouds is sparsely scattered, while the clouds have greater thickness.

Forward Cloud Generator.The function of forward cloud generator is generate the cloud droplets $x$ which satisfy distribution according to known digital characterization Ex, En, and $\mathrm{He}$.

The algorithm steps of one dimensional of forward cloud generator are as follows:

1)Producing a normal distribution, the expectation for $E n$, and the variance is $H e^{2}$, then a normal random number $E n$ ' is extracted.

2)Producing a normal distribution, the expectation is $E n$, and the variance is $E n^{, 2}$, then a normal random number $x$ is extracted.

3)Calculating the certainty $\omega=\exp \left[-(x-E x)^{2} / 2 E n^{, 2}\right], x$ is a cloud droplet value, which is determined to satisfy the above digital characteristics of cloud distribution.

4)Operating Continuously step 1) to 3), which can be pre-specified number of cloud droplets, so far out of the loop.

\section{Assessment Method of Harmonic Emission Level Based on Pan-normal Harmonic Impedance Cloud}

Basic Principal.To simplify the analysis, power system and the user can be equivalent to the circuit shown in Figure 1. The figure shows that the harmonic voltage and harmonic current at PCC are $V_{P C C}$ 
and $I_{P C C}$, respectively; The system side is represented by an equivalent harmonic current source $I s$ and the equivalent of a harmonic impedance $Z s$; The user side is represented by an equivalent harmonic current source $I c$ and the equivalent of a harmonic impedance $Z c$.

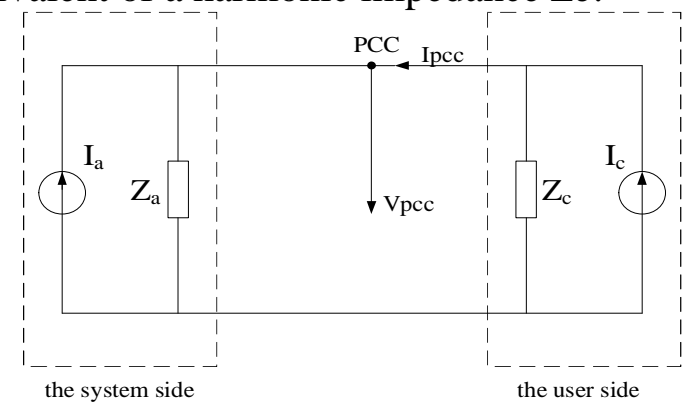

Figure 1.The equivalent circuit for harmonic analysis

In Figure 1, According to the circuit structure on the left side of the dashed box within the system side, the equation can be deduced:

$\Delta V_{P C C}=\Delta I_{P C C} Z_{s}+\Delta I_{s} Z_{s}$

" $\Delta$ " indicates vector difference between the two sample points at the neighborhood time, i.e. the wave of momentum. Similarly, according to the circuit structure on the right side of the dashed box within the user side, the equation can be deduced:

$$
\Delta V_{P C C}=-\Delta I_{P C C} Z_{c}+\Delta I_{c} Z_{c}
$$

The formula (1), (2)are divided by the $\Delta I_{P C C}$ at the same time, then finishing equation can be obtained:

$$
\begin{gathered}
\frac{\Delta V_{P C C}}{\Delta I_{P C C}}=Z_{s}+\frac{\Delta I_{s} Z_{s}}{\Delta I_{P C C}} \\
\frac{\Delta V_{P C C}}{\Delta I_{P C C}}=-Z_{c}+\frac{\Delta I_{c} Z_{c}}{\Delta I_{P C C}}
\end{gathered}
$$

From equation (3) it can be seen, when the system side harmonic currents remain unchanged (i.e. $\Delta I_{s}=0$ ), the fluctuation ratio is equal to $Z s$; Similarly, from equation (4) it can be seen, when the user side harmonic currents remain unchanged (i.e. $\Delta I_{c}=0$ ), the fluctuation ratio is equal to $-Z_{c}$. In the actual power system, the fluctuation of the system side and the user side is not a single one, which is often accompanied by each other. Therefore, the judgment of $\left|\Delta V_{P C C} / \Delta I_{P C C}\right|$ closer to which side is the key to the use of fluctuation ratio to calculate harmonic impedance.

In most practical engineering, although the value of the user harmonic impedance is limited, it is often much larger than the system side, that is, $Z c>>Z s$. Therefore, this paper proposes the use of cloud model to filter out the small fluctuations ratio sample relatively, which is close to the system harmonic impedance, thereby estimating the system harmonic impedance.

Calculate the Digital Features of Impedance.There are several factors interfering harmonic sources, as these factors are independent and random, then it can be proved that the real and imaginary parts of fluctuation ratio of PPC meet the statistical characteristics of generalized Cauchy distribution, while the Cauchy distribution can be seen as a special $t$ distribution and freedom degree is $n$, when $n$ is large enough (in fact, as long as $n \geq 45$ ), the t distribution approximates a standard normal distribution ${ }^{[6]}$. Thus, the real and imaginary parts of the fluctuation ratio of the statistical characteristics can be regarded as approximate normal distribution, that is pan-normal distribution, which satisfy the applicable conditions of the normal cloud model .

Through the above analysis, the pan normal cloud impedance is established according to the real and imaginary parts of the harmonic fluctuation ratio at PCC, then Ex, En and $\mathrm{He}$ are extracted from the cloud droplets by backward cloud generator. The algorithm steps are as follows:

(1)Calculating the sample average $\bar{X}=\frac{1}{n} \sum_{i=1}^{n} x_{i}$

(2)Calculating a first sample absolute central moment $B_{1}=\frac{1}{n} \sum_{i=1}^{n}\left|x_{i}-\bar{X}\right|$

$$
S^{2}=\frac{1}{n-1} \sum_{i=1}^{n}\left(x_{i} \overline{85} \bar{\gamma}\right)^{2}
$$


(3)Calculating the sample variance

(4)Calculating the expectation of normal cloud, entropy and hyper entropy, $E x=\bar{X}, \quad E n=\sqrt{\frac{\pi}{2}} \times B_{1}$

Determine the Impedance Threshold.Ex, En, and He of the impedance cloud can be obtained by 3.2. Through the "3En'rule" of the cloud model to determine the screening threshold,upper and lower thresholds are $\mathrm{Ex}-3(\mathrm{En}+3 \mathrm{He}), \mathrm{Ex}+3(\mathrm{En}+3 \mathrm{He})$ respectively. Thus, the real part $\operatorname{Re}_{(\mathrm{k})}$ and imaginary part ) of the impedance which is selected have to meet the following conditions.

$$
\begin{aligned}
& E x-3(E n+3 H e) \leq R e_{(k)}\left(I_{(k)}\right) \\
& \leq E x+3(E n+3 H e)
\end{aligned}
$$

$\operatorname{Re}_{(k)}$ (or $\left.\operatorname{Im}_{(k)}\right)$ is the real part (or imaginary part) of the fluctuation ratio of the $k$-th. In (5) as a criterion, it can filter out the relatively large fluctuation ratio which is close to the user harmonic impedance, and screen the fluctuation ratio samples closer to the true value which is closer to the system harmonic impedance, the $Z s$ estimated is a good way to restrain the influence of the background harmonic fluctuation and reduce error.

The selection of $\mathrm{He}$ and $\mathrm{En}$ in (5) direct impact the screening effect of the "3En' rule". The greater the $\mathrm{He}$ and $\mathrm{En}$, the more the fluctuation ratio samples which meet filtering condition, but the more information of the user harmonic impedance,resulting in $Z s$ estimation error seriously.Therefore, in

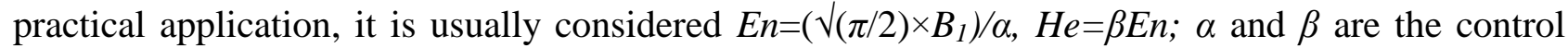
parameters, respectively, 0.29 and 0.1 .

Estimation of the Harmonic Emission Level.Disconnecting the PCC, and the system no-load, the voltage of PCC is $V_{P C C}$ ' at this time; After accessing to the load the voltage of the PCC is $V_{P C C}$ '". According to figure 1, the equation can be listed:

$$
\begin{aligned}
V_{P C C}{ }^{\prime} & =Z_{s} I_{s} \\
V_{P C C} " & =Z_{s}\left(I_{s}+I_{P C C}\right)
\end{aligned}
$$

The harmonic emission level of the PCC is $V_{P C C}$ ' before the user access to system, and the harmonic emission level of the PCC is $V_{P C C}$ "' after the user access to system. The user harmonic emission level can be expressed as the amount of change of harmonic emission level before and after the user access to the PCC, difference between $V_{P C C}$ ' and $V_{P C C}$ '. Therefore, finishing (6), (7) can be obtained formula:

$$
V_{c-P C C}=Z_{s} I_{P C C}
$$

System harmonic emission level as follow:

$$
V_{s-P C C}=Z_{s} I_{s}=V_{P C C}-V_{c-P C C}
$$

\section{Simulation Analysis of Experimental Circuit}

The algorithm is verified by digital simulation based on the equivalent circuit of Figure 1, and matlab is a tool software. A sample point is obtained each minute(one minute to get a harmonic measurement at PCC ), resulting in 250 hours of data, and that is 15000 sample points. The corresponding parameters of the Norton equivalent circuit are shown in Table 1, where $M(M=15000)$ represents the total number of samples $(i=1,2,3, \ldots, 15000) . k(k=0.1,0.5,0.7,0.8,0.9,1.0,1.1,1.4)$ represents relative fluctuation between $I_{s}$ and $I_{c .} \quad R_{I c}$ and $R_{I}$ are independent of each other, and meet the random distribution [-0.2,0.2], [-0.05,0.05], respectively. 
Tab.1 Parameters of Norton circuit

\begin{tabular}{|c|c|c|c|c|c|c|c|c|c|}
\hline \multicolumn{10}{|c|}{ harmonic current source of two sides } \\
\hline \multirow{2}{*}{\multicolumn{2}{|c|}{$I_{c}(i)$}} & \multicolumn{4}{|c|}{ amplitude(A) } & \multicolumn{4}{|c|}{ angle $\left({ }^{\circ}\right)$} \\
\hline & & \multicolumn{4}{|c|}{$143.93 \times[1+R I c(i)]$} & \multicolumn{4}{|c|}{0} \\
\hline & & \multicolumn{4}{|c|}{$k \times 143.93 \times[1+R I s(i)]$} & \multicolumn{4}{|c|}{$70 \times\left[1+0.2 \times \sin \left(180^{\circ} \times i / M\right)\right]$} \\
\hline \multicolumn{10}{|c|}{ harmonic impedance of two sides } \\
\hline & & \multicolumn{4}{|c|}{ amplitude $(\Omega)$} & \multicolumn{4}{|c|}{ angle $\left(^{\circ}\right)$} \\
\hline \multirow{2}{*}{\multicolumn{2}{|c|}{$\begin{array}{l}Z c(i) \\
Z s(i)\end{array}$}} & \multirow{2}{*}{\multicolumn{4}{|c|}{$\begin{array}{c}1 \times\left[1+R_{Z c}(i)\right] \\
{\left[1+0.2 \times \sin \left(180^{\circ} \times i / M\right)\right]}\end{array}$}} & \multirow{2}{*}{\multicolumn{4}{|c|}{$54 \times\left[1+0.1 \times \sin \left(180^{\circ} \times i / M\right)\right]$}} \\
\hline & & & & & & & & & \\
\hline \multicolumn{10}{|c|}{ Tab.2 Estimation errors of $|Z s|$} \\
\hline \multirow{3}{*}{ e.g } & \multirow{3}{*}{$k$} & \multicolumn{8}{|c|}{ estimation error of $|Z s|$} \\
\hline & & \multicolumn{3}{|c|}{ average error/(\%) } & & \multicolumn{4}{|c|}{ maximum error/(\%) } \\
\hline & & 1 & 2 & 3 & 4 & 1 & 2 & 3 & 4 \\
\hline 1 & 0.1 & 1.92 & 1.05 & -0.02 & 0.01 & 5.56 & -3.41 & -0.63 & -1.25 \\
\hline 2 & 0.5 & 9.53 & -1.29 & 0.21 & -0.20 & 20.86 & 10.67 & 2.38 & -3.63 \\
\hline 3 & 0.7 & 11.63 & 1.94 & -0.24 & -0.71 & 26.84 & 12.16 & -3.86 & -5.55 \\
\hline 4 & 0.8 & 12.62 & 3.85 & -0.40 & -0.61 & 30.66 & 18.55 & -3.61 & -6.99 \\
\hline 5 & 0.9 & 13.61 & 6.75 & -0.47 & -1.18 & 34.80 & 21.83 & -4.45 & 6.40 \\
\hline 6 & 1.0 & 14.51 & 10.52 & -0.72 & -1.40 & 37.81 & 27.89 & -4.06 & -6.24 \\
\hline 7 & 1.1 & 16.62 & 14.81 & -0.60 & -0.75 & 40.34 & 33.67 & 6.69 & 10.59 \\
\hline 8 & 1.4 & 19.44 & 21.73 & -2.25 & -2.98 & 51.00 & 54.17 & -7.85 & 12.54 \\
\hline
\end{tabular}

Tab.3 Estimation errors of $95 \%$ probability values of $V_{c-P C C}$

\begin{tabular}{cccccc}
\hline & \multirow{2}{*}{$k$} & \multicolumn{4}{c}{$95 \%$ probability value error of $V_{c-P C C}$ (theoretical value is $\left.17.94 \mathrm{~V}\right) /(\%)$} \\
\cline { 2 - 6 } & & 1 & 2 & 3 & 4 \\
\hline 1 & 0.1 & -3.07 & -1.21 & -2.21 & -2.20 \\
2 & 0.5 & 10.87 & 1.61 & -2.16 & -2.40 \\
3 & 0.7 & 14.81 & 6.30 & -2.83 & -2.45 \\
4 & 0.8 & 15.11 & 9.90 & -2.80 & -3.13 \\
5 & 0.9 & 16.68 & 13.65 & -2.41 & -2.69 \\
6 & 1.0 & 18.50 & 16.03 & -4.06 & -5.06 \\
7 & 1.1 & 19.68 & 20.76 & -3.53 & -4.36 \\
8 & 1.4 & 22.45 & 32.28 & -4.37 & -5.47 \\
\hline
\end{tabular}

Changes in $k$ reflects the different backgrounds harmonic fluctuations; For each case, 60 sample points are used as a sliding window, and 4 methods are used to analyze 15000 sample(method 1 is based on the symbol identification of fluctuation; method 2 is binary regression; method 3 is dominant fluctuation; method 4 be in this article), and the results are shown in table 2 and table 3 . From the results of the table, the calculation error of the symbol identification and the binary regression are increasing rapidly with the increase of $k$. The calculation error in this article is close to the dominant fluctuation, and both of them remain stable with the variable of $k$, and are far smaller than the first two. Especially background harmonic is large, while the method in this article can accurately calculate the system harmonic impedance.It proved that the method in this article can effectively suppress the influence of background harmonics.

\section{Practical Engineering Application Verification}

The actual measurement data from a $150 \mathrm{kV}$ bus, the system short circuit capacity is $7500 \mathrm{MVar}$. The PCC point corresponds to a 100-MVA industrial DC arc furnace, and the measurement data are measured by the LEM TOPAS 1000 series power analyzer with $6400 \mathrm{~Hz}$ sampling frequency. Figure 2 is the 3rd harmonic voltage and current amplitude waveform in a continuous 10 hours at PCC. 


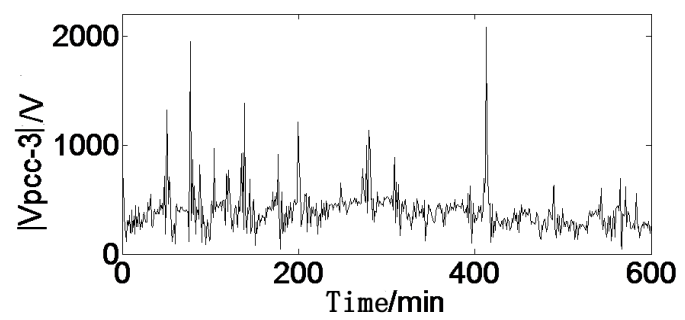

(a)the 3rd harmonic voltage amplitude

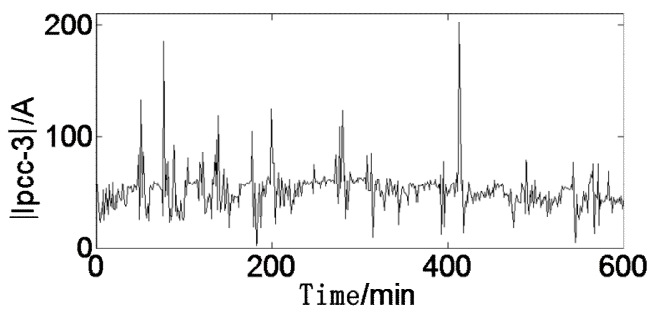

(b)the 3rd harmonic current amplitude

Fig.2 Magnitude waveform of the 3rd harmonic voltage and current at the PCC.

It can be obtained fluctuation ratio by calculation the harmonic current and voltage at PPC, using the cloud model "3En' rule" to filter, filter the results shown in Figure 3. The horizon axis represents the value of the real part of the fluctuation ratio, and the vertical axis represents the value of the imaginary part of the fluctuation ratio, while data oval dashed box is screened qualified fluctuation ratio sample.

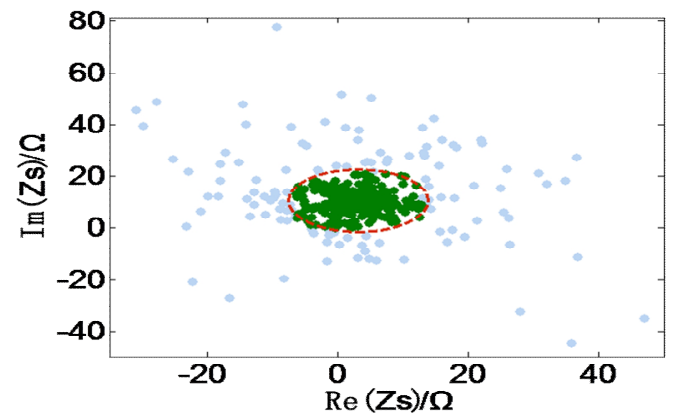

Fig.3 Selection results of the 3rd harmonic Zs

The system harmonic impedance is calculated by using the qualified fluctuation ratio of sample , and the results are compared with the above-mentioned 3 methods, as shown in Figure 4 . Figure 4 is the system harmonic impedance, which is calculated by the $60 \mathrm{~min}$ sliding time window on the $3 \mathrm{rd}$ harmonic data of PCC to reflect the harmonic impedance changes continuously within 10 hours. The method 1 (symbol distinguishing method) to get the impedance magnitude too large, impedance angle is too small, which is due to the real part of the impedance of positive and negative as the sample selection criteria, reserved for the real part is positive but close to the harmonic impedance of user sample, excluding the real part is negative but close to the system harmonic impedance of the sample; Method 2 (binary regression method) affected by fluctuations in the background harmonic results in local instability, and impedance amplitude calculation is relatively small, because the Regression Model in the intercept term can not be treated as constant; Method 3 (dominant fluctuation method) by using the Nair test method to extract the harmonic current fluctuation dominated by the user, in order to estimate the harmonic impedance, effectively suppress the influence of the background harmonic, and the estimated results are stable; Method 4 (in this paper) calculation results and method 3 the closest, with good stability, combined with the third section of the simulation analysis, verify the accuracy of this method.

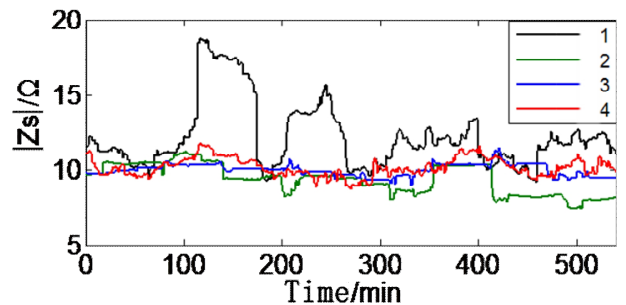

(a)the impedance amplitude

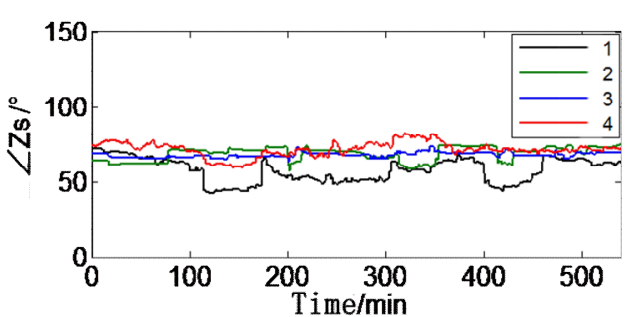

(b)the impedance angle

Fig.4 The estimation of the 3rd harmonic Zs 
Finally, the harmonic voltage emission levels within 10 hours of PCC can be calculated by the equation (8) and (9), the emission level of 95\% probability values as shown in Table 4 , the system harmonic emission level shown in Figure 5.Table 4 and figure 5 further verify the accuracy and effectiveness of the proposed method.

Tab.4 95\% probability values of harmonic emission levels of the two sides

\begin{tabular}{lcc}
\hline & $V_{c-P C C} / \mathrm{V}$ & $V_{s-P C C} / \mathrm{V}$ \\
\hline Method 1 & 1003.41 & 583.53 \\
Method 2 & 769.86 & 238.74 \\
Method 3 & 761.37 & 217.97 \\
Method 4 & 763.54 & 218.39 \\
\hline
\end{tabular}

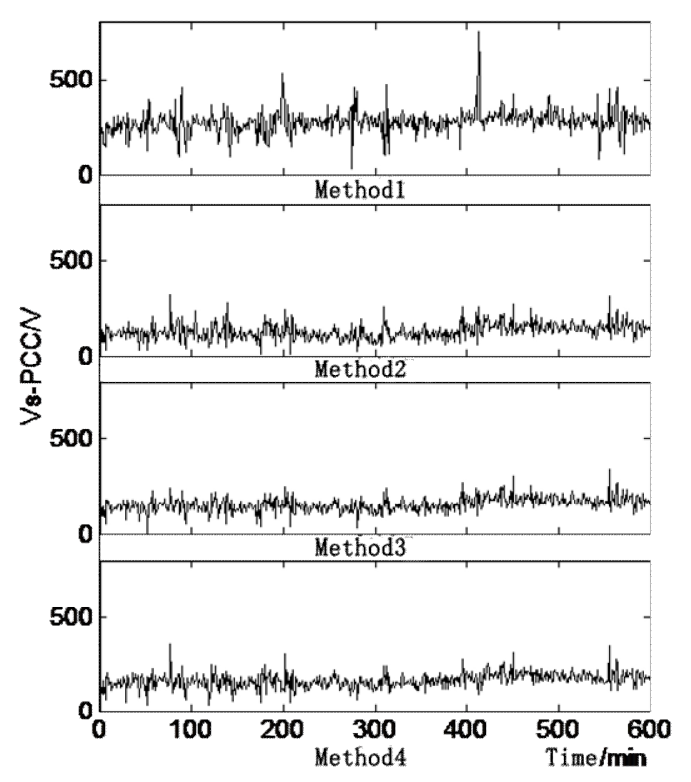

Fig.5 Estimated Vs-PCC based on the 4 methods

\section{Conclusion}

1) Based on the fluctuation method, the probability distribution of the fluctuation ratio is studied, and proposes a new method to estimate system harmonic impedance and harmonic emission level based on the pan-normal impedance cloud.

2) Pan-normal impedance cloud can be established according to the harmonic fluctuation ratio at PCC, extraction the digital features of impedance cloud to determine the filtering threshold, which can effectively identify fluctuation ratio samples closer to the true value through theoretical analysis. The results estimated can restrain the influence of background harmonic fluctuation and improve the estimation accuracy.

3)Through the simulation of theoretical model and calculation of practical engineering examples, it proves the accuracy and effectiveness of the proposed method, and it has a good theoretical research value and engineering application prospect. How to effectively identify remains to be further research at both side of the harmonic impedance less.

\section{References}

[1] Srinivasan K. On separating customer and supply side harmonic contributions[J]. IEEE Transactions on Power Delivery, 1996, 11(2):1003-1012.

[2] Yang H, Pirotte P, Robert A. Harmonic emission levels of industrial loads statistical assessment[C]//CIGRE Proceedings. Paris: International Council on Large Electric 
Systems, 1996:36-306.

[3] Che Quan, Yang Honggeng. Assessing the harmonic emission level based on robust regression method[J]. Proceedings of the CSEE, 2004, 24(4):39-42(in Chinese).

[4] Wei Xingqiong, Zhou Yongquan, Huang Huajuan. Adaptive particle swarm optimization algorithm based on cloud theory [J]. Computer engineering and applications, 2009, 45(1):48-50, 76(in Chinese).

[5] Chen Guilin. Uncertain model of qualitative/ quantitative information transformation — cloud model[J]. Application Research of Computers, 2010, 06:2006-2010(in Chinese). 